\title{
Package Contents Authoring and Consuming System Based on MPEG-21 Multimedia Framework
}

\author{
Nam-Hoon Kim, Min-Jae Kang and Hoe-Kyung Jung, Member, KIMICS
}

\begin{abstract}
Recently there are so many media contents created and used by variety routes. Therefore, it is essential to make a new platform which is used for distributing media contents include IPTV. With open IPTV service, developing the new platform became even more important. Because open IPTV makes it possible for consumers to choose contents regardless of contents of the providers.

The new platform has to accept current variety service structure. MPEG-21 multimedia framework was developed to satisfy these needs. It presented the distribution framework of the contents but didn't restrict the particular solutions for protecting the rights.

In this paper, we present authoring and consuming system for package contents based on MPEG-21 Multimedia Framework for distributing media resources and managing the license of multimedia contents
\end{abstract}

Index Terms - A MPEG-21, Content Authoring, IPTV, Consuming System, Framework

\section{INTRODUCTION}

OPEN IPTV environment is expected to serve advanced services which can make up existing closed IPTV. Open IPTV features a kind of open service by independent contents and devices platform. Independent platform of open IPTV means a concept which includes old DTV broadcast and other contents[1].

In other words, open IPTV has the joinable service structure compromising the close IPTV provider and user created contents(UCC), which means the consumer is also the provider. And independent device platform make it possible to free itself from having service through set-top box(STB) and receive IPTV service using personal computer, ultra-mobile PC(UMPC) and portable multimedia player(PMP).

For these services, it is essential to prepare the new platform which makes possible to implement interoperable authoring and consuming tools by variety contents authors and distribute these contents[2].

\footnotetext{
Manuscript received December 8, 2010; revised January 13 2011; accepted January 19, 2011.

Nam-Hoon Kim is with the Department of Computer Engineering, PaiChai University/KEIT, Daejeon, 302-735, Korea (Email: nhk@keit.re.kr)

Min-Jae Kang is with the Department of Computer Engineering, PaiChai University, Daejeon, 302-735, Korea (Email: kmj5228@ gmail.com)

Hoe-Kyung Jung(Corresponding Author) is with the Department of Computer Engineering, PaiChai University, Daejeon, 302-735, Korea (Email: hkjung@pcu.ac.kr)
}

In this paper, we propose package contents authoring and consuming system to create or play package contents based on MPEG-21 multimedia framework. Also, license server is presented to manage the license information of package contents.

\section{RELATED WORKS}

\subsection{MPEG-21 Multimedia Framework}

The standardization strategy of MPEG-21 defines the standard that describes the technique of protection and managements of contents when the digital contents are delivered to end-user. To define these standards, they conduct the standardization by subdividing in 7 basic parts consisted of Digital Item Declaration(DID), Digital Item and Description(DII), Content handling and usage, Intellectual property management and protection(IPMP), Terminals \& Networks, Content representation, Event Reporting [3,4].

MPEG-21 business model is defined as figure 1 for distributing media contents[5].

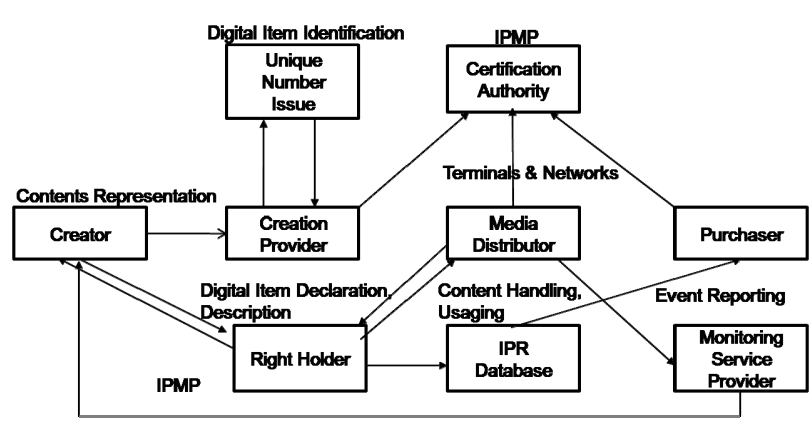

Fig. 1 MPEG-21 Multimedia Framework

\subsection{MPEG-21 Intellectual Property Management and Protection (IPMP)}

When the Digital Item is handled on a diversity of networks and terminals during their life cycle, MPEG-21 offers the safety by protecting and managing the right of contents constantly.

MPEG-21 IPMP's standardization targets are the way of searching IPMP tool and exchanging message between IPMP tools. To achieve this goal, MPEG-21 IPMP creates IPMP component that protect the resource from a third party's access by encapsulating existing DIDL elements [6]. 
IPMP's standardization scope is only how the schema and involving tools that are used to protect the resource are delivered. In other words, they don't restrict the type of tools, so IPMP system guarantees the interoperability of DRM tools, encryption tools and decryption tools developed before IPMP. And it can describe and control the rights of resource using REL and Rights Data Dictionary(RDD)[7,8].

\section{DESIGN OF THE SYSTEM}

In this Section, We present the system architecture and service scenario. There are authors and consumers of package contents as an actor. And license server, package contents authoring \& consuming tools, TV-Anytime User Preference Metadata Manager and package contents provide server are major elements of the system. Finally, we describe the general scenario and real-time streaming $\&$ VOD download service scenario.

\subsection{Major Elements of System}

\subsubsection{Consumer}

A consumer who consumes the granted contents using package contents consuming tools registers license server to request the rights of contents.

\subsubsection{License Server}

License server manages the rights of any content and the information of all members (Consumer, Contents Provider and Contents Author). When the consumer requests any content, license server links the consumer with the contents provide server. Finally, it also offers contents description that registered by authors to the consumer. A license made by license server has the rights information about play times, play, copy, print etc.

\subsubsection{Package Contents Provide Server}

Package Contents Provide Server gains REL file from License Server and packages license file and A/V media file to serve consumer. In this time, package contents file can be served on two ways of VOD or live streaming. To support live streaming broadcast, we built the Package Contents Provide Server as streaming server.

\subsubsection{Package Contents Authoring Tool}

This tool packages $\mathrm{A} / \mathrm{V}$ media and license of consumer. The output file format only can be played by Package Contents Consuming Tool. It registers the tools used for packaging the resources at Tool Server.

\subsubsection{Tool Server}

The tools used in the course of packaging the $\mathrm{A} / \mathrm{V}$ media and license are registered at Tool Server for interoperability between Package Contents Consuming Tools. If the consumers don't have any tool needed to play the package contents, they can download the tool from Tool Server. In this paper, Tool Server also manages the encoder/decoder and Package Contents Consuming Tool.

\subsubsection{Package Contents Consuming Tool}

This tool is used to consume package contents consisted of $\mathrm{A} / \mathrm{V}$ media and a license. It analysis the license to judge whether the consumer has the right or not. If there is no tool to consume the contents, it requests that tool to Tool Server.

\subsubsection{TV-Anytime User Preference Metadata Manager}

We use TV-Anytime schema to control user preference information. When a user requests specific contents, the information that includes genre, play times and run time is recorded in this manager's database. This data can be converted User Preference schema from TVAnytime and be shared with contents providers. So the user can receive more interested contents by this manager. If the user doesn't want to record preference information, this information cannot be recorded to protect the user's privacy according to the TV-Anytime specification [8].

\subsection{Design of Package Contents Authoring Tool}

Package Contents Authoring Tool's main role is packaging the $\mathrm{A} / \mathrm{V}$ media resource and metadata and creates new package contents file. The users also create metadata format using this tool. Created package contents by this tool only can be played specific Package Contents Consuming Tool to manage the rights of resource and user's privacy. Figure 2 describes the design of this tool.

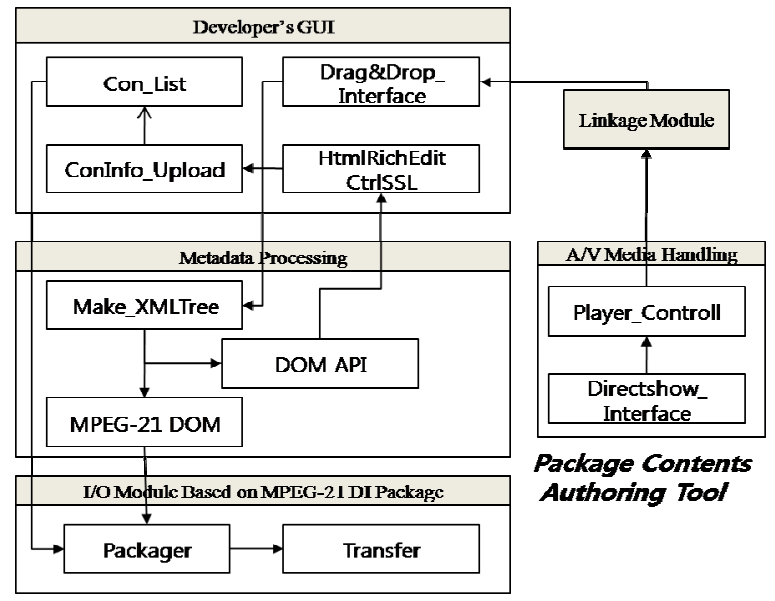

Fig. 2 The Design of Authoring System

\subsubsection{GUI Module for Developer(Author)}

This module make the developer control all modules in Package Contents Authoring Tool and render the metadata information in the form of tree. The developer can create and modify the metadata. Also, all changes 
are sent to each module so that the change is reflected at once.

\subsubsection{I/O Module Based on MPEG-21 DI Package}

This module is consisted of MPEG-21 DI Parser and Metadata Output Section. MPEG-21 DI Parser offer XML/XML Schema parsing, validation check and rendering the metadata in the form of tree through the GUI.

Metadata Output Section output the modified package contents in the type of specific file format which is recommended by MPEG-21 Multimedia Framework. It can raise the interoperability.

\subsubsection{A/V Media Handling Module}

This module is consisted of $\mathrm{A} / \mathrm{V}$ Media Renderer and A/V Media Library. A/V Media Renderer accesses any $\mathrm{A} / \mathrm{V}$ media and renders it to support the preview function of package contents for developer. A/V Media Library plays a role as a repository of $\mathrm{A} / \mathrm{V}$ media files.

\subsubsection{Metadata Processing Module}

Metadata Processing Module is consisted of Metadata Renderer and Metadata Editing Controller. Metadata Renderer does the validation check of the metadata by referencing MPEG-21 DI DOM objects on real-time. The developer can search, access and modify the metadata by Metadata Editing Controller using MPEG-21 DOM API. And we select WYSIYG interface for helping nonprofessional users.

\subsubsection{Linkage Module A/V Media and Metadata}

This module parses the location of the $\mathrm{A} / \mathrm{V}$ media so that A/V Media Handling Module can handle the media files. Also, it supports passing the information of media to the Metadata Processing Module.

\subsection{Design of Package Contents Consuming Tool}

The consumer can receive appropriate service using Package Contents Consuming Tool that offers a series of functions to play the package contents according to the rights described in the metadata. Also, the user can get the information of package contents and its metadata. And Digital Item list that come from package contents provider server can be displayed on display device. This tool has following parts - User GUI Module, I/O Module, MPEG-21 DI Management Module, DID Engine Module, Media Handling Module. This structure is described next Figure 3.

\subsubsection{User GUI Module}

The users can control all of the modules offered by Package Contents Consuming Tools. Main functions are playback controller (play, stop, resize etc) and searching and inquiry the package contents list (Digital Item list).

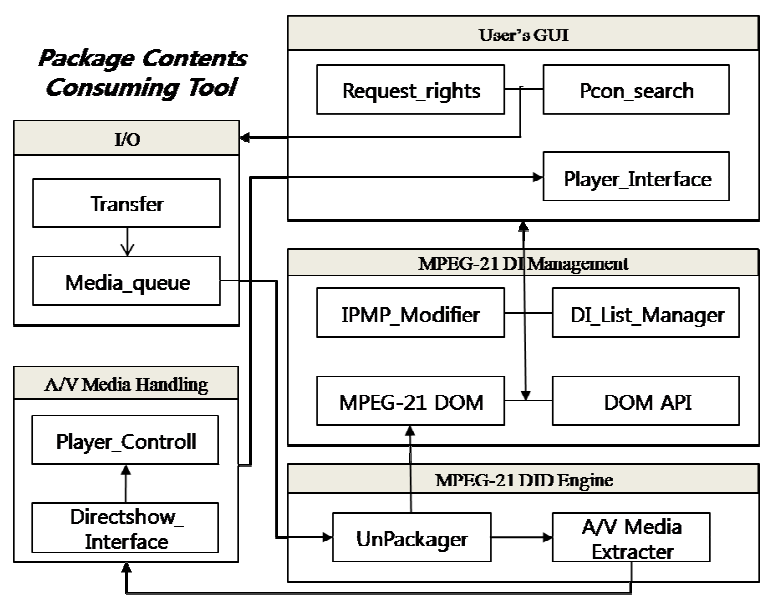

Fig. 3 The Design of Consuming System

\subsubsection{I/O Module}

$\mathrm{I} / \mathrm{O}$ Module works as a network interface, which receives package contents from Package Contents Provide Server and the information needed to play that contents. These data are sent to MPEG-21 DI Management Module. In this point, the information is package contents itself and descriptions of media file (video-audio codec, file size, resolution, playing time).

Moreover, package contents list can be received by I/O Module. This information may help consumers select the contents. In other word, the output section sends the information of selected contents to Package Contents Provide Server. As a result of these courses, the user and provider can communicate each other interactively.

\subsubsection{MPEG-21 DI Management Module}

First of all, MPEG-21 DI Management Module unpackage the MPEG-21 DI received from I/O Module into A/V media and metadata based on MPEG-21 DID. And each data is sent to the Media Handling Module and MPEG-21 Engine Module respectively. Lastly this module sends each MPEG-21 DI to User GUI Module to offering contents information for users. MPEG-21 DI means packaged data of the $\mathrm{A} / \mathrm{V}$ media and the metadata.

Also, the parsed data from the DID Engine Module is returned this module to be offered to the users.

\subsubsection{MPEG-21 DID Engine Module}

MPEG-21 DID Engine Module parses the metadata gained from MPEG-21 DI Management Module to take involving descriptions. In this process, this module supports validation check. The description data is sent or not according to the result of validation check. If the result is not valid, error message may occur.

\subsubsection{Media Handling Module}

Media Handling Module sends the A/V media to User GUI to render the contents and offers playback control functions. Ahead of this course, this module analysis play-rights described in metadata to reflect the right when 
the user request to play package contents.

\section{IMPLEMENTATION}

In this section, interfaces of package contents authoring and consuming system are described. This system is implemented using IBM-PC, Windows XP Service Pack3, and Microsoft Visual Studio 6.0. And MSXML 4.0 SDK is used to parse the XML document.

\subsection{Package contents authoring system}

Package contents authoring system is constructed of two tabs which are information of package contents and management of uploaded contents. Figure 4 is an interface of information of c contents.

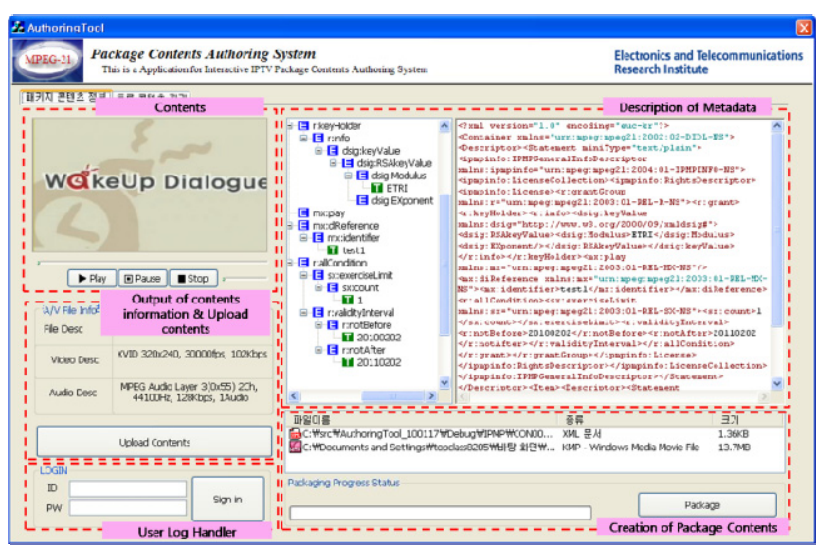

Fig. 4 The interface of Authoring System

\subsubsection{Contents player}

Contents player can play $\mathrm{A} / \mathrm{V}$ media resources before packaging them. We can play $\mathrm{A} / \mathrm{V}$ media resource by selecting a file added on the list in the part of authoring of packing contents.

4.1.2 Output of contents information \& Upload contents

This part outputs the information of video and audio for A/V media file. Also it provides an interface to upload contents on license server.

\subsubsection{User Log Handler}

This part provides an interface to register package contents, obtain the rights for playing and confirm the users of package contents authoring system when they upload the contents.

\subsubsection{Description of Metadata}

This part describes the metadata for inserting into package contents. It is constructed of tree control and rich edit control. The tree control represents the metadata by a form of tree view. The rich edit control represents the context of the metadata.

\subsubsection{Creation of Package Contents}

This part lists the metadata files and $\mathrm{A} / \mathrm{V}$ media files for package contents, packages those files and uploads the package contents on the license server.

\subsection{Package Contents Consuming System}

Package contents consuming system has the contents player, file manager and the interface for requesting rights. The consumers can play the package contents using contents player and file manager. Also, it is possible to request and renew the right for using some package contents. The interface for rights is represented in figure 5.

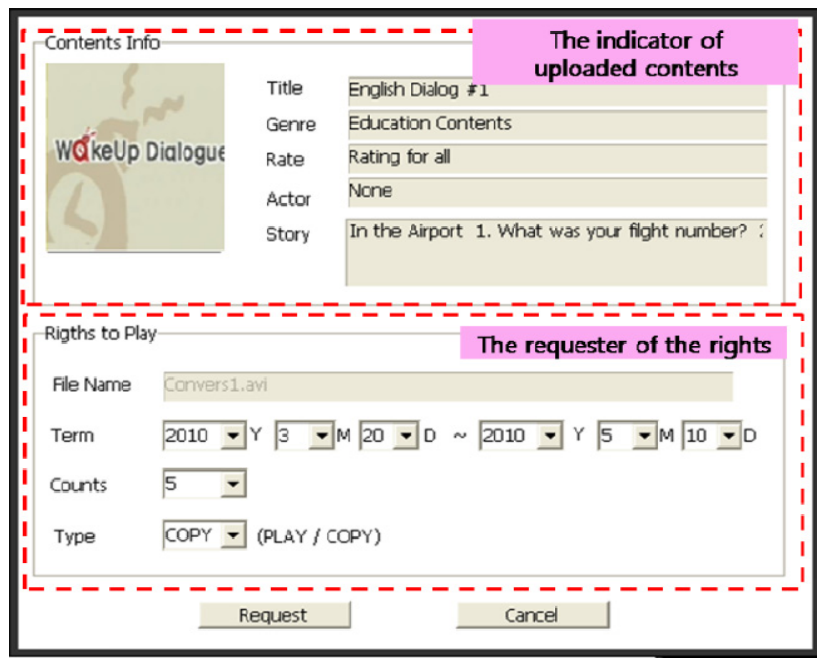

Fig. 5 The interface of Consuming System

\subsubsection{The indicator of uploaded contents}

This part indicates the information of contents which is inputted when those contents were uploaded.

\subsubsection{The requester of the rights}

This part is an interface for requesting the rights of contents and renewing the rights. The items that can be set are the period of play, the number of play and the type of play.

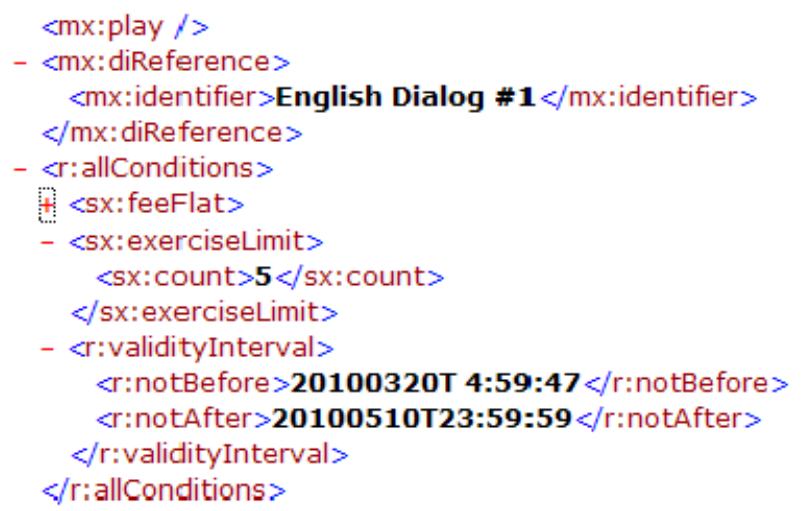

Fig. 8 Metadata of License Information 
The metadata of license information is presented as figure 8 . It is made from the requester of the rights part and transferred to license server system. This metadata is packaged with media contents by authoring system.

\section{CONCLUSIONS}

This paper proposes the framework based on MPEG-21 multimedia framework for distributing the package contents using authoring and consuming system. And the systems for authoring and consuming the package contents are implemented.

Also, we design and implemented $\mathrm{A} / \mathrm{V}$ media packaging module to handle license problems based on MPEG-21 IPMP. Because MPEG-21 IPMP doesn't designate the specific license tools, each service providers can use existing license tools and stat the tools they use. Therefore the consumers can use variety of contents.

Features of this system are as follows. Because we design this system based on MPEG-21 standard, it is interoperable among all systems that adopt the MPEG-21 Specification. So the users will have opportunities to receive more interested contents as compared with the previous platform. Finally, our system can serve real-time streaming and VOD download service as well.

There are some further works related with encryption of license information and performance of streaming server.

\section{REFERENCES}

[1] "Open IPTV Forum", http:// www.openiptvforum-.org.

[2] "DRM Forum", http://ww.drm.or.kr.

[3] ISO/IEC 2100-2 DID Second edition - Part2 : Digital Item Declaration", N6927, Hong Kong, China, 2005.

[4] "Second Study of the Digital Item Identification FCD (ISO/IEC FCD 21000-3)”, N4815, May 2002.

[5] S.H.Son, "Multimedia Framework Standard: MPEG-21", EtriTrend, Dec. 2002.

[6] “ISO/IEC 21000-4 FCD IPMP Components", N7196, April 2005.

[7] "ISO/IEC 21000-5 FCD Rights Expression Language", Dec 2002. [8] "ISO/IEC 21000-6 Rights Data Dictionary (RDD), N4943, July 2002.

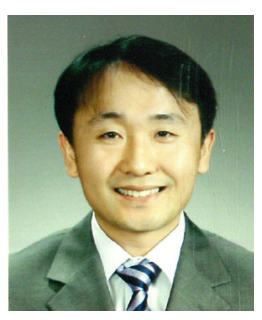

Nam-Hoon Kim He received the B.S. and M.S. degrees in Optical Engineering from Cheongju University, in 1998 and 2000, respectively. From 2000 to 2009, he had worked as a Senior Researcher at Korea Institute of Industrial Technology Evaluation and Planning (ITEP). Since 2009, he has worked as a Senior Officer Korea Evaluation Institute of Industrial Technology (KEIT). His current research interests include XML, USN, Web Service.

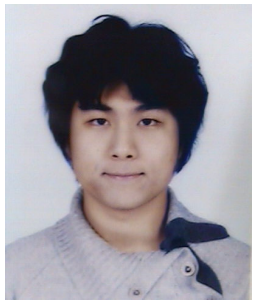

Min-Jae Kang He received the B.S. degrees in computer engineering from PaiChai University, in 2011. He is master course students of department of computer of PaiChai University. His current research interests include XML, USN and EDI system.

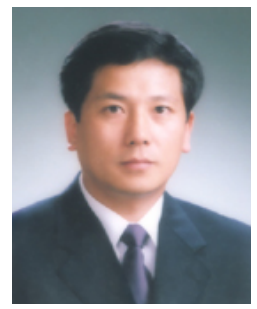

Hoe-Kyung Jung He received the B.S., M. S. and $\mathrm{Ph} . \mathrm{D}$. degrees in computer engineering from Kwangwoon University, in 1985, 1987 and 1993, respectively. Since 1994, he has worked as a professor in Department of Computer Engineering at PaiChai University. His current research interests include XML, semantic web, MPEG-21, Mobile RFID 